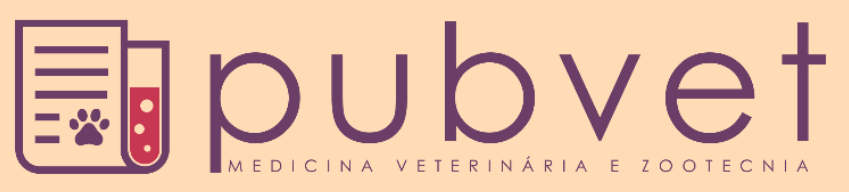

https://doi.org/10.31533/pubvet.v16n02a1041.1-8

\title{
Princípios gerais da ortodontia em cães: Revisão
}

\author{
Lucas Tiago Bomfim ${ }^{1 *}$, Iam Ramos de Souza ${ }^{1}$, Sabrina Rodrigues de Medeiros Dias ${ }^{1}$, Kaique \\ Leite de Oliveira Santos ${ }^{1}$
}

${ }^{I}$ Graduado (a) em Medicina Veterinária pela Universidade do Grande Rio Prof. José de Souza Herdy - Unigranrio, campus Duque de Caxias, RJ, Brasil.

*Autor para correspondência, E-mail: lucas.veterinario.rj@gmail.com

\begin{abstract}
Resumo. O objetivo deste trabalho foi realizar uma revisão de literatura sobre os princípios gerais da ortodontia em cães, a fim de fornecer uma referência para veterinários interessados neste tipo de tratamento. $\mathrm{O}$ atendimento odontológico é necessário para proporcionar uma boa saúde e melhorar a qualidade de vida dos animais, pois as doenças da cavidade oral não tratadas são dolorosas e podem contribuir para doenças locais e sistêmicas. A razão de qualquer tratamento ortodôntico em animais é aliviar a dor da má oclusão e restaurar a função dos dentes, não sendo realizada por motivos estéticos. A escolha do método de tratamento deve ser baseada na idade e temperamento do cão, no custo do tratamento, na capacidade do dono de aderir ao protocolo de tratamento como mentor e na habilidade e conhecimento do dentista veterinário em usar o aparelho e metodologia adequada para cada caso. Considera-se que uma oclusão dentária normal é fundamental para a saúde e conforto do animal. As má-oclusões podem ter origem hereditária ou adquirida e seu diagnóstico deve ser feito o mais cedo possível, para isso a cooperação do tutor é fundamental para atingir os resultados desejados.
\end{abstract}

Palavras-chave: Aparelho ortodôntico, má-oclusão dentária, medicina veterinária

\section{General principles of orthodontics in dogs: Review}

\begin{abstract}
The aim of this work was to carry out a literature review on the general principles of orthodontics in dogs, in order to provide a reference for veterinarians interested in this type of treatment. Dental care is necessary to provide good health and improve the quality of life of animals, as diseases of the untreated oral cavity are painful and can contribute to local and systemic diseases. The reason for any orthodontic treatment in animals is to alleviate the pain of malocclusion and restore tooth function, not being performed for aesthetic reasons. The choice of treatment method should be based on the dog's age and temperament, the cost of treatment, the owner's ability to adhere to the treatment protocol as a mentor, and the veterinarian dentist's skill and knowledge in using the appropriate device and methodology for each case. It is considered that a normal dental occlusion is essential for the health and comfort of the animal. Malocclusions can have a hereditary or acquired origin and their diagnosis must be made as soon as possible. For this, the tutor's cooperation is essential to achieve the desired results.
\end{abstract}

Key words: Orthodontic appliance, dental malocclusion, veterinary medicine

\section{Principios generales de la ortodoncia en perros: Revisión}

Resumen. El objetivo de este trabajo fue realizar una revisión de la literatura sobre los principios generales de la ortodoncia en perros, con el fin de proporcionar una referencia para los veterinarios interesados en este tipo de tratamientos. El cuidado dental es necesario 
para brindar una buena salud y mejorar la calidad de vida de los animales, ya que las enfermedades de la cavidad bucal no tratadas son dolorosas y pueden contribuir a enfermedades locales y sistémicas. El motivo de cualquier tratamiento de ortodoncia en animales es aliviar el dolor de la maloclusión y restaurar la función de los dientes, no realizándose por motivos estéticos. La elección del método de tratamiento debe basarse en la edad y el temperamento del perro, el costo del tratamiento, la capacidad del propietario para adherirse al protocolo de tratamiento como mentor y la habilidad y el conocimiento del dentista veterinario en el uso del dispositivo y la metodología adecuados para cada caso. Se considera que una oclusión dental normal es fundamental para la salud y comodidad del animal. Las maloclusiones pueden tener un origen hereditario o adquirido y su diagnóstico debe realizarse lo antes posible, por lo que la colaboración del tutor es fundamental para lograr los resultados deseados.

Palabras clave: Aparato de ortodoncia, maloclusión dental, medicina veterinaria

\section{Introdução}

A má-oclusão é um problema dentário comum em cães domésticos que pode causar perda ou restrição da função mastigatória e lesões se estiverem em contato com os tecidos orais ou com outros dentes. Pode ser ocasionada por vários fatores, como retenção de dentes decíduos, dentes supranumerários, padrões de erupção irregulares, disparidade no tamanho da mandíbula e relação desproporcional entre o dente e o tamanho da mandíbula (Carvalho, 2008; Oakes \& Beard, 1992; Polkowska et al., 2014).

A maioria das más oclusões são problemas hereditários que resultam da reprodução seletiva de outras características, como tamanho do corpo, focinho mais curto ou nariz mais longo (DuPont, 2005). A classificação geral distingue entre má-oclusão congênita e adquirida. Os defeitos congênitos ocorrem no período embrionário, como resultado de fatores genéticos. Em contraste, os defeitos adquiridos se desenvolvem durante a vida pós-natal e resultam de fatores ambientais, como hábitos deletérios e traumatismos. Antes do tratamento, a causa do distúrbio, a idade do paciente, bem como o tipo e a gravidade da anormalidade devem ser determinados (Carvalho, 2008; Polkowska et al., 2014).

A American Association of Orthodontics, define a ortodontia em humanos, como o ramo da odontologia responsável pela supervisão, controle e correção das estruturas dentofaciais, bem como alterações que requerem o movimento dos dentes. No entanto, o principal objetivo da ortodontia em animais é melhorar as condições de vida do paciente para proporcionar bem-estar (Ciffoni \& Pachaly, 2001).

Em vários países os princípios éticos das diversas associações determinam que não é ético realizar intervenções ortodônticas para fins estéticos em animais, pois alterações na morfologia do animal por esses motivos são consideradas mutilações (Carvalho, 2008; Ciffoni \& Pachaly, 2001). O tempo de tratamento em animais varia do tipo de aparelho necessário, com média de um a seis meses (Carvalho, 2008; Ciffoni \& Pachaly, 2001; Mendoza, 2020).

O objetivo deste trabalho foi realizar uma revisão de literatura sobre os princípios gerais da ortodontia em cães, abordando a anatomia do dente, classificação das má-oclusões, sinais clínicos, tipos de aparelhos ortodônticos, movimentação ortodôntica, tratamentos e seus cuidados, a fim de fornecer uma referência para veterinários interessados neste tipo de tratamento devido à sua importância.

\section{Anatomia do dente}

Os dentes são estruturas que se encontram localizadas nos alvéolos dos ossos incisivos, do osso maxilar e da mandíbula. Os cães nascem edentados e por volta das duas a quatro semanas erupcionam os dentes decíduos, num total de 28. A perda destes dentes começa aos 3-5 meses de idade e, com 5-8 meses, erupciona a dentição definitiva constituída por 42 dentes que são divididos em quatro grupos (Figura 1) de acordo com a função, sendo eles, os incisivos, os caninos, os pré-molares e os molares, tendo como função prender, cortar, dilacerar e triturar os alimentos, respectivamente. Algumas variações nas idades de dentição podem ser causadas por saúde, nutrição, sexo ou raça (Amimoto et al., 1993; Carvalho, 2008; Gouveia, 2009; Niemiec, 2021; Silva, 2016). 
Apesar da variação do tamanho, da forma e da função, a composição e estrutura dos dentes são semelhantes (Logan, 2006). O dente é anatomicamente composto pela coroa, corresponde à porção acima da linha da gengiva sendo coberta pelo esmalte e não é inervada nem vascularizada, e pela raiz, constituída pela porção abaixo da linha da gengiva que funciona como âncora. Os tecidos que compõem o dente são o esmalte, dentina, cemento e polpa dentária (Figura 2) (Gouveia, 2009; Logan, 2006; Silva, $\underline{2016}$.

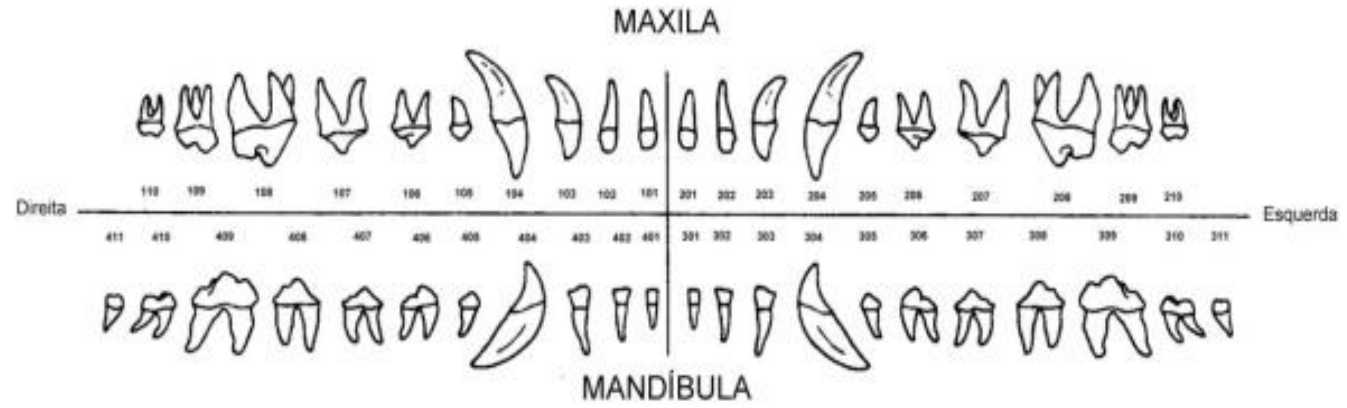

Figura 1. Odontograma do cão. Fonte: Silva (2016).

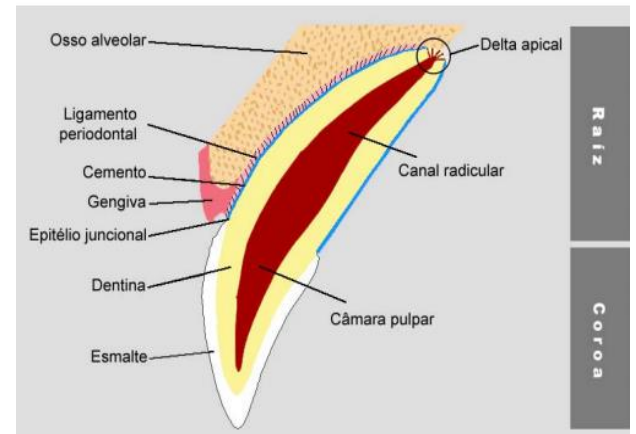

Figura 2. Anatomia dentária de um cão. Fonte: Gioso (2007)

A coroa do dente é protegida por uma fina camada de esmalte. A espessura do esmalte em cães varia de aproximadamente $0,1 \mathrm{~mm}$ a $1 \mathrm{~mm}$. A polpa dentária é um tecido mole que contém vasos sanguíneos, nervos (apenas sensoriais) e outros tipos de células, incluindo odontoblastos, fibroblastos e fibrócitos. A dentina é organizada em túbulos, que se estendem da polpa até a área onde a dentina e o esmalte se encontram (junção dentino-esmalte) ou a raiz onde o cemento e o esmalte se encontram (junção cemento-esmalte). Os túbulos dentinários são preenchidos por fluidos, e são extensões citoplasmáticas dos odontoblastos. Esses túbulos são os principais caminhos para a difusão do material na dentina, um conceito conhecido como permeabilidade dentária. Devido à relação interdependente entre a polpa e a dentina, esses dois tecidos são mais bem considerados como uma formação única: o complexo dentinapolpa. A polpa e a dentina funcionam como uma unidade porque os odontoblastos, que revestem a parede da cavidade pulpar, projetam suas extensões na dentina. Além disso, os odontoblastos são responsáveis pela formação da dentina. Por sua vez, o tecido pulpar depende da dentina para proteção (Niemiec, 2014).

O ligamento periodontal (LP) é constituído por fibras de tecido conjuntivo denso (colágeno), que são conhecidos como fibras de Sharpey. Essas fibras se fixam ao cemento e ao osso alveolar através das quais o dente se sustenta no alvéolo e em estado contínuo de atividade fisiológica (Silva, 2016).

\section{Classificação das má-oclusões}

Os defeitos de mordida são comuns na prática clínica de pequenos animais, principalmente em cães. Para diagnosticar defeitos na oclusão existe uma série de fatores nos quais o médico veterinário deve se concentrar durante a avaliação do paciente, pois existem particularidades anatômicas com fenótipo diferente, dependendo da raça do animal. Além disso, existem conjuntos de alterações que podem ser admitidos por alguns padrões raciais (Carvalho, 2008; Ciffoni \& Pachaly, 2001). 
Silva (2016) relatou em seu estudo que existem inúmeras propostas de classificação para as máoclusões e o sistema mais frequentemente utilizado é o da classificação segundo Angle, mas que em Medicina Veterinária, utiliza-se a classificação de Angle modificada, a qual se divide em cinco classes (Tabela 1).

Uma avaliação ortodôntica completa, assim como a funcionalidade do sistema estomatognático, deve avaliar as situações dolorosas da cavidade oral resultantes de má-oclusões. Dentes com oclusão imprópria dentro da boca podem não exercer suas funções de forma eficaz (Carvalho, 2008)

Tabela 1. Classificação das má-oclusões segundo Angle

\begin{tabular}{lll}
\hline Classe & \multicolumn{2}{c}{ Características } \\
\hline Classe 0 & Ortoclusão & $\begin{array}{l}\text { A oclusão normal está descrita como a perfeita interdigitação entre os dentes } \\
\text { maxilares e mandibulares. }\end{array}$ \\
\hline Classe I (MOC I) & Neutroclusão & $\begin{array}{l}\text { Não existe alteração na relação do comprimento de mandíbula e da maxila. } \\
\text { Má-oclusão dentária }\end{array}$ \\
\hline Classe II (MOC II) & Distoclusão & $\begin{array}{l}\text { O arco dentário superior encontra-se em posição rostral ao arco dentário } \\
\text { inferior. Má-oclusão esquelética }\end{array}$ \\
\hline Classe III (MOC III) & Mesioclusão & $\begin{array}{l}\text { O arco dentário superior apresenta-se caudamente em relação ao arco dentário } \\
\text { inferior. Má-oclusão esquelética. }\end{array}$ \\
\hline Classe IV (MOC IV) & Assimétricas & $\begin{array}{l}\text { Apresenta alterações mistas. Não é considerada como uma verdadeira classe } \\
\text { pela American Veterinary Dental College (AVDC). Classificada como } \\
\text { máoclusão assimétrica ou wrybite. }\end{array}$ \\
\hline
\end{tabular}

Fonte: Silva (2016).

\section{Sinais clínicos}

As má-oclusões podem ter origem hereditária (como o caso do prognatismo e braquignatismo) ou adquirida (resultante de fraturas e luxações mal resolvidas) e podem ocasionar problemas físicos e de comportamento (Moraillon et al., 2013).

O mau posicionamento dos dentes pode levar cães a desenvolverem desgastes e fraturas dentárias, lesões na cavidade oral, formação de tártaro e cárie, desenvolvimento de doença periodontal, alteração no desenvolvimento dento facial e artrose temporo-mandibular, gerando sensibilidade, inflamação, focos infecciosos que podem evoluir para abscessos, com isso ocorre à manifestação dos sinais clínicos das afecções como anorexia, perda de peso, ptialismo, mudanças na preensão e mastigação do alimento, desconforto e odor fétido. Em quadros mais avançados também se observam sinais comportamentais das enfermidades orais como aflição e angústia, escavar o chão ou coçar a área afetada, balançar a cabeça e friccioná-la contra obstáculos, comportamento agressivo (causado por dor crônica), seleção de alimento na dieta, corrimento oronasal, alimento não digerido nas fezes (Carvalho, 2008; Gioso, 2007; Moraillon et al., 2013; Polkowska et al., 2014).

\section{Aparelhos ortodônticos}

Na prática clínica médico-veterinária tem sido utilizado diversos tipos de aparelhos ortodônticos, porem os fixos são os mais indicados (Oakes \& Beard, 1992). Destes, três modelos são utilizados mais frequentemente, sendo eles: o de plano inclinado, construído em resina acrílica ou compósito e utilizado para a correção da lingualização dos caninos (Hale, 1996; Pavlica \& Cestnik, 1995), o parafuso expansor, uma técnica em que a força exercida na movimentação dentária provém da ativação de um pequeno parafuso metálico inserido numa placa de resina e fixado sobre os dentes (Niemiec, 2014) e os brackets, que são peças metálicas fixadas nos dentes com resina composta fotopolimerizável, que após a fixação, são colocadas as correntes elásticas para se obterem as forças adequadas que consigam o deslocamento dos dentes envolvidos (Niemiec, 2014).

A intervenção ortodôntica em animais se baseia em três classificações, ortodontia preventiva, interceptativa e a ortodontia corretiva. A ortodontia preventiva é utilizada para prevenir a má oclusão antes mesmo que ela ocorra, como realizar a extração de dentes decíduos antes que provoquem mesialização nos dentes definitivos. A ortodontia interceptativa, quando usamos forças passivas para guiar a erupção, visando eliminar condições que determinam irregularidades no desenvolvimento e maturação da oclusão, enquanto os ossos e dentes ainda estão em crescimento. A ortodontia corretiva baseia-se no princípio da movimentação dentária, onde os dentes terminaram sua erupção, pela ação de 
forças diretas provenientes, geralmente, da utilização de aparelhos fixos sobre as peças dentárias (Niemiec, 2014; Mendoza, 2020).

Ao usar aparelhos ortodônticos na prática clínica, deve-se sempre lembrar de usar a quantidade de força adequada em relação à direção da movimentação dentária ortodôntica, a fim de evitar danos ao tecido dentário, ao ligamento, necrose do tecido periodontal, atrofia horizontal do processo alveolar, reabsorção radicular e perda dentária. As forças utilizadas no tratamento ortodôntico não devem causar deslocamento de um dente maior que cerca de $0,1 \mathrm{~mm}$ por dia (Polkowska et al., 2014).

O significado de força em ortodontia é determinado como a ação exercida por um corpo (parafuso, elástico, mola ou arame), em cima de outro corpo (osso, palato ou dente). A força é demonstrada em massa vezes aceleração. Além disso, é um vetor que apresenta: a Intensidade medida em gramas; um direcionamento que pode ser inclinado ou reto, possuindo um ângulo entre a linha e um eixo de referência; e um módulo que é a tendência da força (Mendoza, 2020). Uma força também é definida como uma ação com magnitude e direção. A unidade com a qual a força é medida é dada em Newtons $(\mathrm{N})$, mas na Ortodontia é utilizada em gramas $(\mathrm{g})$. O fator para converter gramas em Newtons é $1 \mathrm{~g}=$ $0,00981 \mathrm{~N}$, ou $1 \mathrm{~N}=101,937 \mathrm{~g}$. Sendo assim, a força necessária seria entre $20 \mathrm{a} 26 \mathrm{~g} / \mathrm{cm}^{2}$. Na ortodontia medimos a força e resistência de fios e elásticos, com um aparelho chamado tensiômetro, que apresenta duas extremidades para acoplar os elásticos ou qualquer fixação para que, no momento em que sejam acionados, possam medir a força ou intensidade da força que é medida por marcadores que o instrumento possui em uma das pontas (Mendoza, 2020).

\section{Movimentação dentária ortodôntica}

O movimento ortodôntico pode ser definido como a aplicação prolongada de uma pressão controlada sobre o elemento dentário, resultando em movimento do dente e remodelação do osso alveolar (Silva, 2016). Este movimento dentário é considerado pelos médicos ortodontistas como um evento biológico mediado pelas células do ligamento periodontal (LP) (Ferguson \& Wilcko, 2016).

A incidência de carga mecânica altera a homeostasia do ligamento periodontal. A partir do início do período de stress causado pela incidência da força, células de defesa são acionadas, com o objetivo de restaurar a função normal dos tecidos envolvidos com imediata reação no cemento, no ligamento periodontal e no osso alveolar. A massa óssea é determinada pelo equilíbrio entre reabsorção e formação ao longo da vida, em resposta às demandas funcionais. $\mathrm{O}$ osso responde aos estímulos mecânicos por deformação e recomposição de formato e a movimentação ortodôntica ocorre graças à esta propriedade. Remodelação óssea é um processo fisiológico que abrange reabsorção e sobreposição (Lima, 2017).

O sistema biológico do dente reage à variação na magnitude da força, tempo de aplicação que produzem remodelação óssea e movimentação dentária ortodôntica. O movimento dentário pode ocorrer de uma forma rápida ou lenta, dependendo das características físicas da força aplicada e da resposta biológica do ligamento periodontal sendo diferente do movimento dentário fisiológico. A força ideal deve ser suave e contínua, proporcionando movimento rápido sem resultar em danos às estruturas de suporte com mínimo desconforto ao paciente. O osso em contato com a compressão sofre uma reabsorção resultante do processo inflamatório produzido pela força aplicada, e no lado da tensão ocorre uma deposição óssea fazendo com que aquele dente permaneça na nova posição. É necessário um período de retenção até a maturação óssea neoformada estar completa (Krishnan \& Davidovitch, 2006; Li et al., 2018; Silva, 2016). A movimentação dentária errônea ou exagerada dos quartos pré-molares superiores nos tratamentos ortodônticos podem causar rompimento do suprimento vascular e fistulação (Dias et al., 2013).

Devido às consequências associadas ao deslocamento dentário, tais defeitos devem ser diagnosticados o mais precocemente possível, para que as medidas adequadas possam ser tomadas para inibir ou eliminar completamente os defeitos. Pacientes com ossos ainda em crescimento são menos resistentes à movimentação dentária ortodôntica e seus tecidos moles são mais flexíveis. Suas células estão na fase de crescimento ativo e exibem alta adaptabilidade às mudanças em curso. Nessas condições, é mais fácil conseguir movimentar os dentes, sendo a idade de 9 a 15 meses considerada o período mais favorável para o tratamento (Polkowska et al., 2014). 
Segundo Silva (2016) ao avaliar a utilização de aparelhos ortodônticos em 23 cães, relatou que em seu estudo o tempo de permanência do aparelho variou entre 34 a 97 dias. Segundo o trabalho, animais mais jovens requerem um tempo de tratamento inferior e esta diferença também pode estar relacionada com o tipo de movimento pretendido com cada tipo de aparelho para correção das má-oclusões dentárias.

\section{Tratamento e seus cuidados}

O tratamento ortodôntico é indicado quando o animal apresenta má-oclusão dolorosa e desintegradora. É uma alternativa não invasiva e pode impedir a necessidade de extração de dentes importantes. Como resultado do tratamento ortodôntico, a forma das arcadas dentárias e a posição de cada dente podem ser corrigidas, mantendo as relações anatômicas e funcionais adequadas na cavidade oral e nas estruturas circundantes (Figura 3) (Polkowska et al., 2014).

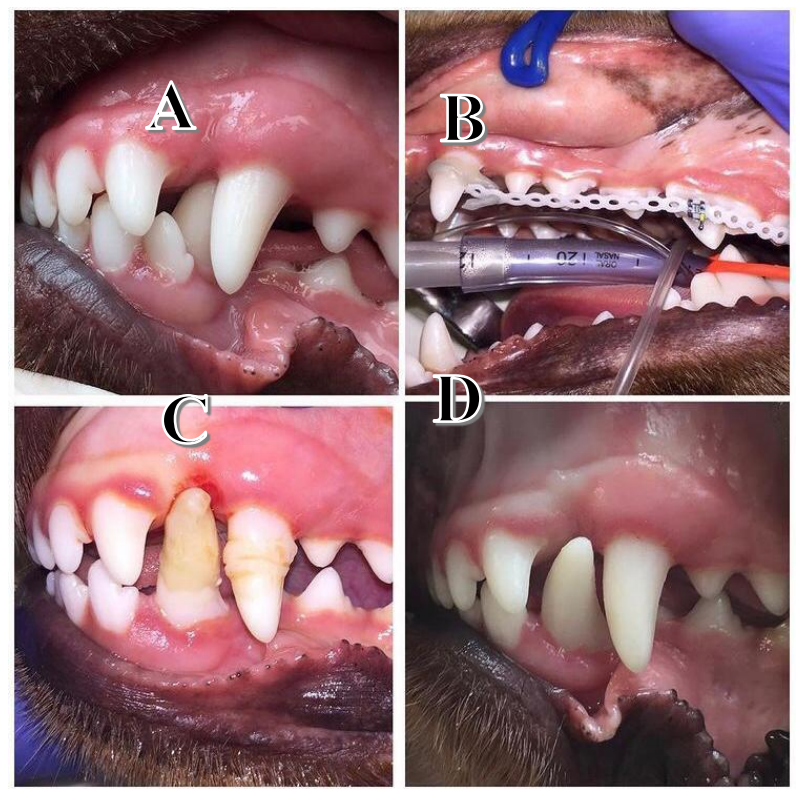

Figura 3. Evolução da correção da má-oclusão em cão. A) fotografia antes do tratamento, apresentando canino inferior $\mathrm{e}$ linguovertido. B) Iniciado o movimento ortodôntico com o objetivo a deslocar caudalmente o canino superior. C) removidos incisivos inferiores e lateralização de canino inferior. D) Tratamento ortodôntico concluído. Fonte: Arcevo de Morten Hinge, 2021

Caso o animal possua alguma bacteremia, seja paciente de alto risco ou que será submetido a outras intervenções cirúrgicas, recomenda-se a administração previa de antibióticos de amplo espectro de ação. É importante poupar a microbiota oral normal, constituída por bactérias Gram positivas, que inibem a colonização por bactérias anaeróbicas Gram negativas e por isso deve-se evitar o uso prolongado de antibióticos. O medicamento pode ser administrado imediatamente antes da intervenção cirúrgica, com alta dosagem e rápida absorção. Os antibióticos mais utilizados são a Clindamicina (6 a $11 \mathrm{mg} / \mathrm{kg}$, BID), Amoxicilina/Clavulanato (15 mg/kg, BID), Enroflaxacina (5 mg/kg, SID), Doxiciclina em animais adultos (3 a $5 \mathrm{mg} / \mathrm{kg}$, BID) e Espiramicina/Metronidazol (75.000 UI $/ \mathrm{kg}-12,5 \mathrm{mg} / \mathrm{kg}$, SID) (Gioso \& Carvalho, 2004).

A avaliação radiográfica é essencial para a observação das estruturas dentárias e dos tecidos adjacentes. Um dos inconvenientes da avaliação radiográfica intraoral é a necessidade de anestesia geral. As radiografias intraorais são preferíveis em relação às radiografias do crânio completo, uma vez que eliminam a sobreposição das diferentes estruturas cranianas e orais e permitem uma maior resolução e constituem um meio de diagnóstico indispensável, na medida em que permitem a avaliação dos dentes e do osso quanto à reabsorção óssea e às afecções endodônticas (Gouveia, 2009). A maior dificuldade na utilização da película radiográfica consiste no posicionamento correto do animal tendo-se de movimentar a sua cabeça. A aquisição de uma unidade radiográfica odontológica elimina essa dificuldade uma vez que pode ser facilmente manobrada. A prática radiográfica intraoral utiliza duas 
técnicas principais, a técnica do paralelismo (Figura 4A) que é usada apenas quando o eixo longo do objeto a ser radiografado, neste caso o dente, e a película se encontram paralelos entre si e a técnica da bissectriz (Figura 4B), usada quando o objeto a ser radiografado e a película radiográfica não estão paralelos entre si, como no caso dos dentes maxilares e dos dentes incisivos e caninos da mandíbula (Gouveia, 2009).

A cooperação do tutor é fundamental, tanto no uso de técnicas onde são necessárias ativações do aparelho em casa ou mesmo nos cuidados com a higienização e, principalmente, na execução de medidas para evitar que o animal danifique o dispositivo, impossibilitando o sucesso do tratamento (Niemiec, 2014). Caso o tutor note sinais de inflamação, ulceração ou corrimento, deve contatar o médico veterinário assim que possível (Wiggs \& Lobprise, 1997). O movimento ortodôntico leva tempo. Levará semanas a meses para atingir o resultado desejado e o sucesso depende de um monitoramento cuidadoso com exames frequentes e possivelmente ajustes sob sedação ou anestesia (미, 1996).

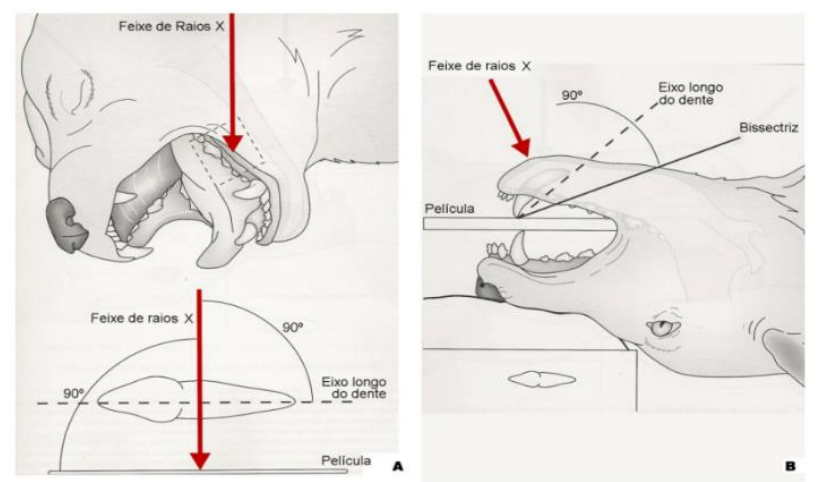

Figura 4. Radiografia intraoral em cães A) Técnica do paralelismo: usada apenas quando o eixo longo do objeto a ser radiografado, neste caso o dente, e a película se encontram paralelos entre si. B) Técnica da bissectriz: usada quando o objeto a ser radiografado e a película radiográfica não estão paralelos entre si, como no caso dos dentes maxilares e dos dentes incisivos e caninos da mandíbula. Fonte: Gouveia (2009).

\section{Considerações finais}

A oclusão dentária normal é essencial na manutenção da saúde oral dos animais, proporcionando melhor qualidade de vida a eles. A oclusões podem ter origem hereditária ou adquirida e devem ser diagnosticadas o mais cedo possível e submetidas à correção com o uso de aparelho ortodôntico adequado, pois animais mais jovens possuem tempo de tratamento mais curto pelo fato do osso alveolar ser menos denso e proporcionar menos resistência ao movimento ortodôntico. A educação do tutor do animal é fundamental para obter o resultado desejado à partir das recomendações de tratamento.

\section{Referências bibliográficas}

Amimoto, A., Iwamoto, S., Taura, Y., Nakama, S., \& Yamanouchi, T. (1993). Effects of surgical orthodontic treatment for malalignment due to the prolonged retention of deciduous canines in young dogs. Journal of Veterinary Medical Science, 55(1), 73-79. https://doi.org/10.1292/jvms.55.73

Carvalho, V. G. G. (2008). Cirurgia ortognática em cães: técnica intra-oral de osteotomia sagital da mandíbula para correção de prognatismo e retrognatismo. Estudo em cadáveres. Universidade de São Paulo.

Ciffoni, E. M. G., \& Pachaly, J. R. (2001). Considerações históricas e legais sobre a odontologia veterinária no Brasil. Arquivos de Ciências Veterinárias e Zoologia Da UNIPAR, 4(1), 49-54.

Dias, F. G. G., Pereira, L. F., Mattos Júnior, E., Rocha, J. R., \& Dias, L. G. G. G. (2013). Fístula infraorbitrária na espécie canina. Centro Científico Conhecer, 10(18), 442-448. 
Dupont, G. (2005). Orthodontics in Pets; Correcting What Can Cause Pain in the Mouth! World Small Animal Veterinary Association World Congress Proceedings. Shoreline Veterinary Dental Clinic, Seattle, WA, USA.

Ferguson, D. J., \& Wilcko, M. T. (2016). Tooth movement mechanobiology: toward a unifying concept. In B. Shroff (Ed.), Biology of Orthodontic Tooth Movement (pp. 11-44). Springer. https://doi.org/10.1007/978-3-319-26609-1_2.

Gioso, M A. (2007). Odontologia em animais selvagens: Curso teórico prático. Associação Brasileira de Odontologia Veterinária.

Gioso, Marco Antônio, \& Carvalho, V. G. (2004). Métodos Preventivos para a manutenção da boa saúde bucal em cães e gatos. Clínica Veterinária, 9(52), 68-76.

Gouveia, A. I. E. A. (2009). Doença periodontal no cão. Universidade Técnica de Lisboa.

Hale, F. A. (1996). Orthodontic correction of lingually displaced canine teeth in a young dog using lightcured acrylic resin. Journal of Veterinary Dentistry, 13(2), 69-73. https://doi.org/10.1177/089875649601300202.

Krishnan, V., \& Davidovitch, Z. (2006). Cellular, molecular, and tissue-level reactions to orthodontic force. American Journal of Orthodontics and Dentofacial Orthopedics, 129(4), 469-e1. https://doi.org/10.1016/j.ajodo.2005.10.007.

Li, Y., Jacox, L. A., Little, S. H., \& Ko, C.-C. (2018). Orthodontic tooth movement: The biology and clinical implications. The Kaohsiung Journal of Medical Sciences, 34(4), 207-214. https://doi.org/10.1016/j.kjms.2018.01.007.

Lima, C. A. C. (2017). Biologia da movimentação dentária: Abordagem de eventos celulares e moleculares microscópicos ocorridos no ligamento periodontal, responsáveis pelos efeitos macroscópicos da movimentação ortodôntica. Revista Científica InFOC, 2(2), 61-70.

Logan, E. I. (2006). Dietary influences on periodontal health in dogs and cats. Veterinary Clinics: Small Animal Practice, 36(6), 1385-1401. https://doi.org/10.1016/j.cvsm.2006.09.002.

Mendoza, G. G. (2020). Ortodoncia veterinaria: Principios básicos del movimento óseo dental. Principios básicos em ortodoncia. . Vanguardia Veterinaria.

Moraillon, R., Legeay, Y., Boussarie, D., \& Sénécat, O. (2013). Manual Elsevier de Veterinária: Diagnóstico e tratamento de cães, gatos e animais exóticos (7th ed.). Elsevier.

Niemiec, B A. (2014). Disorders of dental hard tissus in dogs. Todays,s VeterinartyPratice, 97, 1-7.

Niemiec, Brook A. (2021). Breed predispositions to dental and oral disease in dogs. Wiley Online Library.

Oakes, A. B., \& Beard, G. B. (1992). Lingually displaced mandibular canine teeth: orthodontic treatment alternatives in the dog. Journal of Veterinary Dentistry, 9(1), 20-25. https://doi.org/10.1177/089875649200900104.

Pavlica, Z., \& Cestnik, V. (1995). Management of lingually displaced mandibular canine teeth in five bull terrier dogs. Journal of Veterinary Dentistry, 12(4), 127-129. https://doi.org/10.1177/089875649501200409.

Polkowska, I., Golynska, M., Sobczynska-Rak, A., Putowska, K., Matthews-Brzozowska, T., Szyszkowska, A., Golynski, M., Chelminski, A., \& Capik, I. (2014). Orthodontic treatment of dogs on the basis of modern knowledge and own experience. Bulletin of the Veterinary Institute in Pulawy, 58, 645-650. https://doi.org/10.2478/bvip-2014-0098.

Silva, A. F. C. S. T. (2016). Aparelhos ortodônticos activos e passivos: estudo da sua aplicação em 23 cães. Universidade Lusófona de Humanidades e Tecnologias.

Wiggs, R. B., \& Lobprise, H. B. (1997). Veterinary Dentistry: principles and practice. Lippincott-Raven Publishers.

Histórico do artigo:

Recebido: 30 de setembro de 2021 Aprovado: 30 de outubro de 2021

Disponível online: 28 de fevereiro de 2022
Licenciamento: Este artigo é publicado na modalidade Acesso Aberto sob a licença Creative Commons Atribuição 4.0 (CC-BY 4.0), a qual permite uso irrestrito, distribuição, reprodução em qualquer meio, desde que o autor e a fonte sejam devidamente creditados. 